\title{
Hochfrequenzgeräte, Absaugung, Blutsperre: Anwendungen, Funktionsweisen, Risiken
}

\author{
Fabian Schäfer, Jürgen Degreif
}

\section{Zusammenfassung}

Im modernen Operationssaal kommt eine Vielzahl von technischen Geräten zum Einsatz. $\mathrm{Zu}$ den am häufigsten verwendeten gehören dabei die, die im Wesentlichen für gute Sicht im OP-Gebiet und einen möglichst geringen Blutverlust des Patienten zuständig sind: Hochfrequenzgeräte (zum Koagulieren und Schneiden), Absaugung und Blutsperren. Obwohl sie dem OP-Personal genauso geläufig sind wie Skalpell und Pinzette, ist die Kenntnis ihrer Funktionsweise keineswegs Allgemeingut und sind die Risiken bei ihrer Anwendung nicht immer offensichtlich. Daher soll in diesem Artikel ein Grundverständnis für die technischen Hintergründe vermittelt und aus dem praktischen Alltag auf typische Fallstricke (auch im wörtlichen Sinne!) eingegangen werden.

\section{Geräte der Hochfrequenzchirurgie}

Basis dieser Geräte ist die Verwendung von hochfrequentem Wechselstrom. Im Gegensatz zu den $50 \mathrm{~Hz}$ des Haushaltsstroms kommen dabei Frequenzen von über $300000 \mathrm{~Hz}(=300 \mathrm{kHz})$ zum Einsatz. Bei diesen Frequenzen tritt im Gewebe vor allem ein thermischer Effekt auf, sprich, das Gewebe wird erhitzt. Würde man niedrigere Frequenzen oder Gleichstrom einsetzen, käme es im Wesentlichen zur Reizung von Muskelund Nervenzellen bzw. zu Ionenverschiebungen. Beides ist selbstverständlich unerwünscht.

\section{Anwendungen}

Koagulieren

Durch Koagulieren von Gewebe kann zumindest bei kaliberschwächeren Gefäßen - eine effektive Blutstilllung erreicht werden. Im Vergleich zu Verfahren wie der Ligatur oder dem Einsatz von Fibrinkleber ermöglicht das eine erhebliche Einsparung von Zeit und Kosten. Ideal

OP-JOURNAL 2005; 21: 22-27

(c) Georg Thieme Verlag KG Stuttgart · New York für diesen Zweck ist ein Erhitzen des Gewebes auf ca. $70-100^{\circ} \mathrm{C}$. Dabei verdampft intra- und extrazelluläre Flüssigkeit, Kollagene werden in Glukose umgewandelt und das Gewebe schrumpft. Gefäßwände ziehen sich dabei so weit zusammen, dass Blutungen stehen.

Schneiden

Durch schnelles Erreichen höherer Temperaturen als beim reinen Koagulieren $\left(>100^{\circ} \mathrm{C}\right.$ ) kommt es zum explosionsartigen Zerreißen der Zellmembranen. Gewebe lässt sich auf diese Weise durchtrennen. Vorteile des Verfahrens sind die gleichzeitig erreichte Blutstillung, eine Schnittführung ohne mechanisches Trauma und ohne Kraftaufwand, sowie ein keimabtötender Effekt (allerdings mit dem Nachteil einer Gewebsnekrose behaftet, die per se wieder ein höheres Infektrisiko mit sich bringt).

\section{Techniken}

Sowohl Blutstillung, als auch das Durchtrennen von Gewebe sind mono- oder bipolar möglich. In beiden Fällen ist ein geschlossener Stromkreis nötig; der Unterschied zwischen den Techniken besteht darin, wo sich die beiden Pole des Stromkreises befinden.
Eine aufwändigere Variante für spezielle Indikationen ist die Argonplasma-Koagulation.

\section{Monopolare Technik}

Unter monopolarer Technik versteht man, dass das Arbeitsgerät des Chirurgen den einen Pol des Stromkreises, die so genannte Aktivelektrode, darstellt; der andere Pol wird durch die großflächige Neutralelektrode gebildet, die sich an einer anderen Stelle des Körpers befindet.

Das Prinzip dieses Verfahrens ist, eine hohe Stromdichte an der Spitze des Arbeitsgeräts zu erreichen, so dass sich nur dort ein nennenswerter thermischer Effekt einstellt; an der Neutralelektrode verteilt sich der gleiche Gesamtstrom auf eine viel größere Fläche und kann deshalb - wenn man alles richtig macht - keinen Schaden anrichten. Bei diesem Verfahren fließt der Strom auf einer mehr oder weniger weiten Strecke durch den Patienten und kann dabei z.B. die Funktion eines Herzschrittmachers oder einer EKG-Ableitung beeinträchtigen, wenn diese „im Weg“ liegen.

Die Sicherheit des Verfahrens hängt entscheidend davon ab, wie und wo die Neutralelektrode angebracht wird. Während der Operation ist dort aufgrund der Abdeckung meist keine Sichtkontrolle mehr möglich und Komplikationen werden oft zu spät bemerkt.

Das in der traumatologischen und orthopädischen Chirurgie häufig verwendete Koagulieren mit Klemme oder Pinzette ist nur mit der monopolaren Technik möglich, da hier das Hilfsinstrument als Aktivelektrode dient.

\section{Bipolare Technik}

Hier befinden sich beide Elektroden in der Spitze des Arbeitsgeräts; eine separate Neutralelektrode ist nicht notwendig. Das bedeutet, dass der Strom nur über 
eine sehr kurze Strecke fließt und die Risiken der Neutralelektrode vermieden werden.

Hilfsinstrumente wie Pinzetten können nicht eingesetzt werden, was zwar die Flexibilität einschränkt, andererseits aber das Handling vereinfacht, da eben nur ein Gerät vom Operateur bzw. Assistenten gehalten werden muss.

In der Traumatologie und Orthopädie kommen diese Geräte besonders zur subtileren Blutstillung, wie z. B. in der Handchirurgie, zum Einsatz. In der minimalinvasiven Chirurgie sind sie klar überlegen.

\section{Argonplasma-Koagulation}

Bei diesem Verfahren wird Plasma, d.h. ionisiertes und damit leitfähiges Gas, als Aktivelektrode eingesetzt. Der Strom fließt - ähnlich wie bei der monopolaren Technik - von einer Hochfrequenzelektrode über das Argonplasma durch den Körper und wird von einer Neutralelektrode abgeleitet. Das Gas leuchtet, wodurch der Verlauf des Stroms sichtbar wird. Ohne direkten Gewebskontakt der Elektrode kann so v.a. flächenhaft koaguliert werden.

Beim argongestützten Schneiden wird die Schneideelektrode von Argongas umströmt, das hier jedoch nicht ionisiert ist. Dadurch wird der Schneidebereich von Sauerstoff abgeschirmt, was Verkohlung und Rauchbildung verringert.

Der Anwendungsbereich dieser Verfahren liegt besonders in der - auch laparoskopischen - Abdominalchirurgie und der Endoskopie, derzeit weniger in der orthopädisch-traumatologischen Chirurgie.

\section{Praktischer Einsatz}

\section{Vorbereitung der Operation}

Für die Sicherheit des Patienten entscheidend ist die richtige Lagerung. Er muss isoliert von leitfähigen Gegenständen gelagert werden, besonders wenn sie geerdet sind, wie z.B. OP-Tische, Infusionsständer und ähnlichem. Dies wird v.a. durch zwei Maßnahmen gewährleistet:

- Der Patient liegt komplett auf einem guten Isolator, in der Regel einer speziell dafür vorgesehenen Kunststoffmatte

- Der Patient muss trocken gelagert sein, da praktisch jede Feuchtigkeit elek-

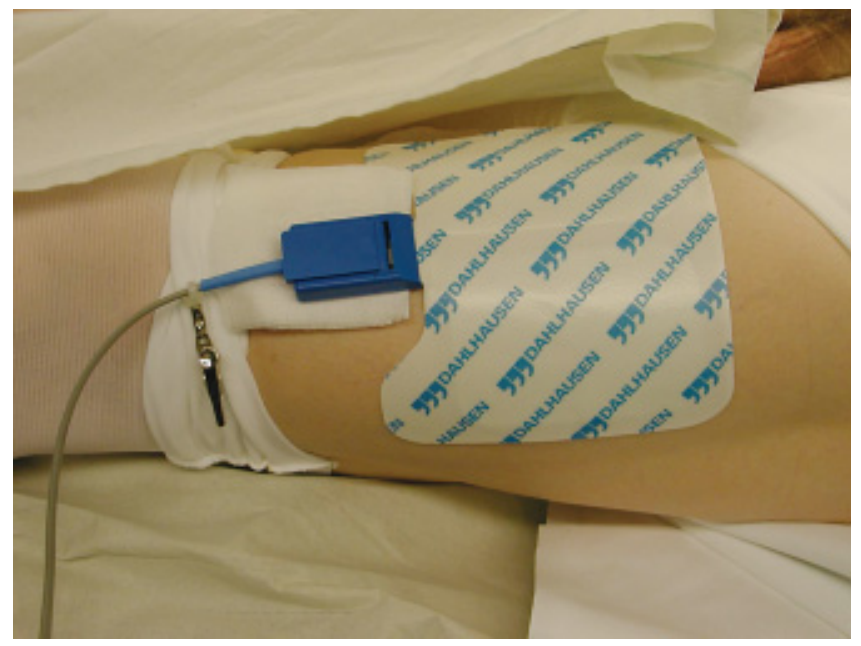

Abb. 1 Typische Platzierung der Neutralelektrode am Oberschenkel; großflächige Elektrode, Haut rasiert.

trisch leitet und eine Verbindung zwischen Patient und z.B. dem OP-Tisch herstellen kann, über die dann unerwünschte Ströme fließen können.

In der Praxis ist der zweite Punkt schwerer über den Gesamtverlauf einer Operation hinweg zu gewährleisten. Blut, Urin, Infusionslösungen etc. können eine anfänglich trockene Lagerung schnell zunichte machen. Intraoperativ ist es dann oft schwierig, an dieser Situation etwas zu ändern.

Daher sollten im Vorfeld einige Dinge bedacht werden:

- Ist mit einem längeren Operationsverlauf zu rechnen (z. B. über 1,5-2 Stunden), sollte ein Blasenkatheter erwogen werden, besonders, falls eine Neutralelektrode verwendet wird und die Ströme durch den Körperstamm fließen (wie bei der Hüftendoprothetik)

- Die Abdeckung sollte unbedingt verhindern, dass Blut oder Spüllösung ihren Weg unter den Patienten finden können (natürlich auch aufgrund hygienischer Erwägungen). Durch Absaugen und Wechseln vollgesogener Unterlagen (steril und unsteril) kann dies zusätzlich verhindert werden.

Beim Verwenden einer Neutralelektrode sind zusätzliche Vorsichtsmaßnahmen nötig. Beim Platzieren der Elektrode auf dem Patienten sollte auf folgende Punkte geachtet werden:

- Elektrode möglichst nah am Operationsgebiet anbringen; typischerweise an Oberarm oder Oberschenkel (Beispiel s. Abb.1)

- Die lange Kante der Elektrode sollte zum OP-Gebiet zeigen; an der kurzen Kante oder einer Ecke der Elektrode entsteht eine deutlich höhere Feldstärke, was zu einem verstärkten thermischen Effekt führt (manche Geräte überwachen die korrekte Ausrichtung, indem sie die Stromverteilung auf der Elektrode messen)

- Die Haut unter der Elektrode muss rasiert und trocken sein, um einen gleichmäßigen Kontakt zu gewährleisten

- Ideal als „Unterlage“ der Neutralelektrode ist Muskulatur, da sie einen geringen elektrischen Widerstand aufweist und somit die thermische Belastung geringer ist (daher die Extremitäten bevorzugen). Wenig geeignet sind Fettgewebe (wie z.B. am Bauch), Knochen, Narbengewebe und unebene Hautregionen. Außerdem sollte darauf geachtet werden, dass keine Implantate unter der Elektrode liegen, da hier ebenfalls eine Erwärmung eintreten kann und zumindest theoretisch Ionen aus Metalllegierungen freigesetzt werden können

- Große Elektroden sind sicherer als kleine, da sie zu einer geringeren Stromdichte führen

- Ausreichenden Abstand (ca. $15 \mathrm{~cm}$ ) von EKG-Elektroden halten

- Herzschrittmacher sollten außerhalb des Stromverlaufs liegen. Im Zweifelsfall Rücksprache mit dem Hersteller oder einem Kardiologen.

Ein weiterer wichtiger Punkt (der übrigens genauso für die Themen Absaugung und Blutsperre gilt) ist das Handling der Kabel. Größtenteils banal, soll dennoch das Wesentliche kurz erwähnt werden:

- Kabel so führen, dass sie im OP-Gebiet nicht behindern und ausreichend Spielraum bieten 
- Der Weg zwischen OP-Tisch und Gerät ist besonders kritisch bezüglich der Sterilität, da es oft keine klare Grenze zwischen sterilem und unsterilem Bereich gibt, besonders wenn die Kabel hoch geführt sind

- Falls vom OP-Tisch abgetreten oder die Position gewechselt werden muss, sind Kabel oft ein Hindernis und sollten ggf. kurz ausgesteckt werden

- Die Befestigung der Kabel im sterilen Bereich ist auf verschiedene Weise möglich (mit Klemmen, Klettverschlüssen, Kompressen o.ä.; s. Abb. 2 als Beispiel für eine einfache und kostengünstige Methode), sollte aber immer ein Verrutschen verhindern

- Kabel sind sehr empfindlich gegen Knicken und Abklemmen.

Sorgfältige Vorbereitung (Lagerung der Patienten, Anbringen von Neutralelektrode etc.) sind entscheidend für die Sicherheit bei der Hochfrequenzchirurgie.

\section{Intraoperativ}

Die intraoperativen Risiken der Hochfrequenzchirurgie sind die offensichtlicheren, da sie in der Regel mit direkt sichtbaren Schäden zu tun haben.

Hauptgefahr ist die überflüssige Gewebstraumatisierung. Sie entsteht, wenn:

- Zu lange koaguliert wird

- Die Leistung des Gerätes zu hoch eingestellt ist (manche Geräte bieten zusätzliche Sicherheit durch automatisches Regulieren der Spannung)

- Besonders beim Koagulieren in der Tiefe mit Pinzetten oder Klemmen diese versehentlich Hautkontakt haben und dort Nekrosen gesetzt werden

- Bei der subkutanen Blutstillung nicht auf genügenden Abstand zur Haut geachtet wird (zur Vermeidung s. Abb. 3).

Weitere Punkte, die zu beachten sind:

- Beim Schneiden und Koagulieren ist durch Absaugen auf Bluttrockenheit an der Instrumentenspitze zu achten - einerseits zur besseren Sicht, andererseits wird sonst der thermische Effekt deutlich reduziert

- Wenn möglich, aufgrund der geringeren Risiken die bipolare Technik verwenden (bei einem Eingriff können selbstverständlich beide Verfahren parallel eingesetzt werden)

n Überflüssiges Koagulieren (und damit Gewebsnekrosen) vermeiden. Viele kleine subkutane Blutungen stehen nach kurzer Zeit spontan

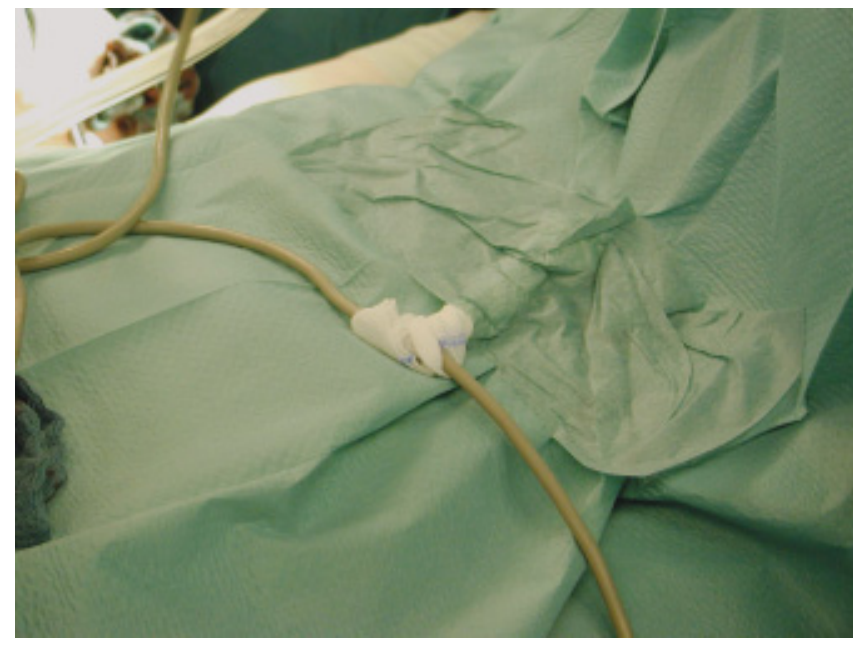

Abb. 2 Beispiel für Kabelbefestigung mit Kompresse und Tape.

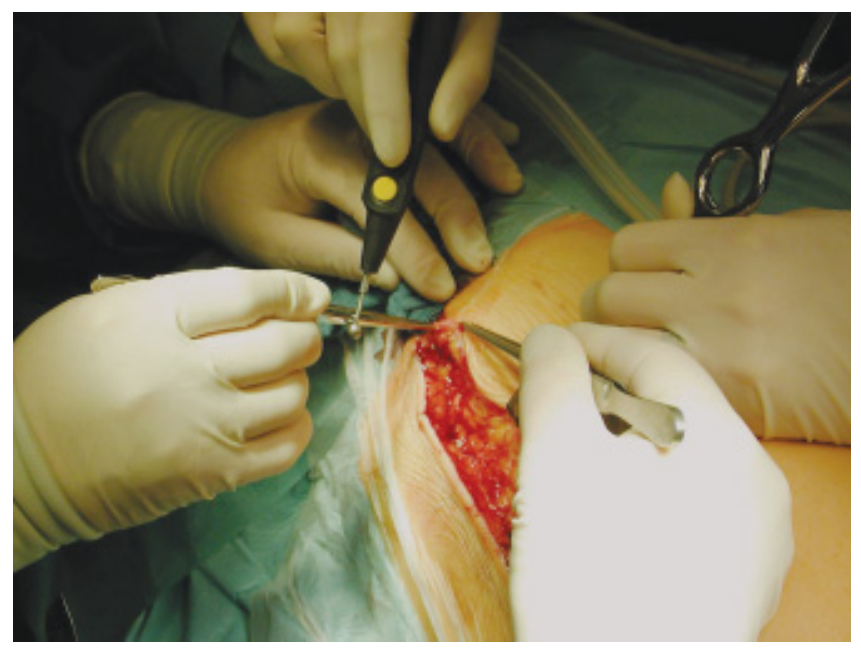

Abb. 3 Beiseitehalten der Haut beim subkutanen Koagulieren - Vermeiden von Hautnekrosen.

Elektrodenspitzen sollten auch intraoperativ immer wieder gereinigt werden (am besten mit einem feuchten Tuch); ansonsten verkohlen Blut und Gewebsreste und wirken als Isolator oder verschmutzen das OP-Gebiet.

Intraoperativ v. a. auf das Vermeiden einer überflüssigen Gewebstraumatisierung achten.

\section{Postoperativ}

Hochfrequenzgeräte müssen regelmäßig kontrolliert und gewartet, die wiederwendbaren Instrumente natürlich gereinigt und sterilisiert werden. Alle Hersteller bieten dazu detaillierte Anweisungen, so dass dieser Punkt hier ausgespart wird.

\section{Absaugung}

\section{Anwendungen}

Geräte zur Absaugung sind unverzichtbar für das Operieren unter guter Sicht. Besonders im tieferen OP-Situs und in Körperhöhlen kann nur so ausreichende
Bluttrockenheit erreicht werden. Auch beim Spülen des OP-Gebietes, von Gelenken etc. ist eine effektive Absaugung entscheidend. Endoskopische Verfahren sind ohne Absaugung undenkbar.

Zunehmend spielt außerdem der Aspekt der Rückgewinnung von Blutbestandteilen des Patienten mit speziellen Systemen eine Rolle. Bei blutungsreichen Operationen (Beispiel Hüftendoprothetik) können Fremdblutgaben vermieden werden, indem körpereigene Erythrozyten aufbereitet und retransfundiert werden.

\section{Funktionsweise}

Alle Saugsysteme arbeiten mit Unterdruck, der auf unterschiedliche Weise erzeugt bzw. bereitgestellt werden kann. In Kliniken steht in der Regel ein hausinternes Leitungssystem für Druckluft und Vakuum zu Verfügung, an das die jeweiligen Geräte angeschlossen werden können. Außerdem gibt es - besonders für den ambulanten Bereich - mobile Sauggeräte, in denen der Unterdruck z. B. mit Kolben/ 


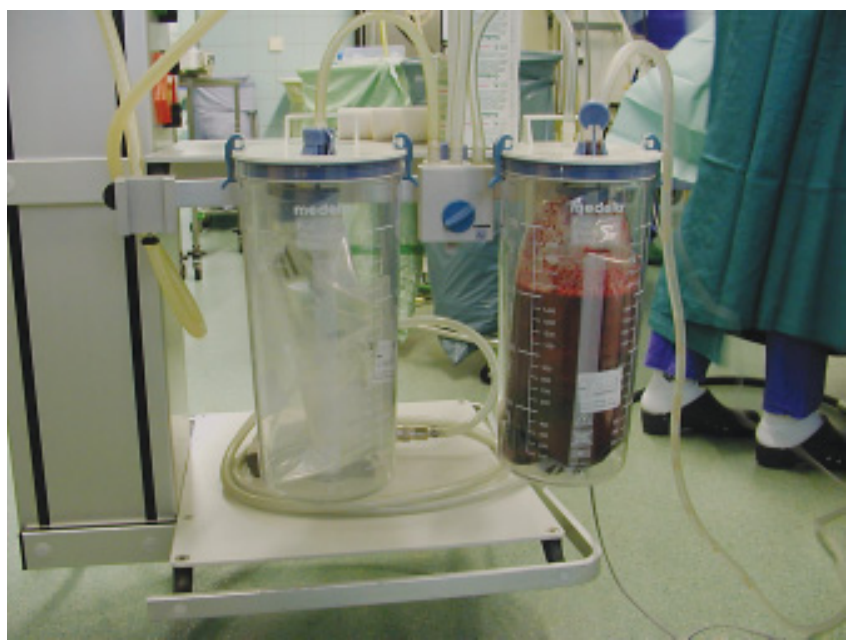

Abb. 4 Beispiel für Sekretauffangbehälter. Zwischen den Behältern kann schnell gewechselt werden.
Zylinder-Systemen erzeugt wird. Je nach Indikation wird mit Unterdrücken von bis zu ca. $-700 \mathrm{mmHg}$ gearbeitet. Dabei können bis zu 50 Liter Luft pro Minute angesaugt werden. Typischerweise werden die Sekrete im OP in Kunststoffbeuteln aufgefangen, die von außen dem Unterdruck ausgesetzt sind und ein Ventil haben, durch das die zunächst enthaltene Luft entweichen kann, welches aber gleichzeitig einen Schutz vor Überlaufen darstellt und mit einem Bakterienfilter versehen ist. Um einen reibungslosen Betrieb zu gewährleisten, sollten wenigstens zwei Systeme parallel vorhanden sein; dann kann, nachdem ein Beutel gefüllt ist, schnell auf den anderen gewechselt werden (s. Abb.4). In den Beuteln können Substanzen vorhanden sein, die das abgesaugte Sekret in einen gelartigen Zustand überführen und so ein hygienisches Entsorgen erleichtern.

Abgesaugt wird mit Spitzen in unterschiedlichen Größen aus Kunststoff oder Metall. Diese können mit einem Verschluss versehen sein, der dem Operateur erlaubt, die Absaugung nur bei Bedarf zu aktivieren. Dies reduziert einerseits die nicht unerhebliche Geräuschbelästigung und verringert andererseits das - allerdings wohl eher geringe - Risiko einer Wundkontamination durch Ansaugen von Luft im OP-Gebiet.

In Systemen, die eine Rückgewinnung von Erythrozyten erlauben, muss das abgesaugte Blut am Gerinnen gehindert werden, daher ist der Absaugschlauch doppellumig, und durch das kleine Lumen fließt eine gerinnungshemmende Lösung (typischerweise Heparin) zur Absaugspitze, um sich dort mit dem Blut zu mischen.

\section{Praktischer Einsatz}

Von den hier besprochenen Geräten ist die Absaugung sicherlich das am wenigsten kritische. Ernst zu nehmende Gefahren bestehen kaum, und es geht eher darum, einen reibungslosen Betrieb aufrechtzuerhalten, um den OP-Ablauf nicht zu stören.

Die beiden häufigsten Gründe für ein Aussetzen des Saugers im Betrieb (und die entsprechenden Maßnahmen) sind:

- Verstopfen der Leitung und der Saugerspitze

- Durchspülen z.B. mit steriler Kochsalzlösung

- Direktes Entfernen von Fremdkörpern z.B. mit der Pinzette

- Absaugbeutel ist voll (subjektiv oft in einem besonders ungünstigen Moment!)

- Rechtzeitiger Wechsel auf leeren Beutel

Beim Entsorgen ist vor allem die Infektionsgefahr für das Personal zu beachten. Das Tragen von Schutzhandschuhen sollte daher bei allen Manipulationen an den Absaugvorrichtungen selbstverständlich sein.

Bei der Absaugung ist v. a. das Hilfspersonal in CP (Springer) für das reibungslose Funktionieren entscheidend.

\section{Blutsperre}

\section{Anwendungen}

Während die beiden oben beschriebenen Geräte sozusagen der Schadensbegrenzung (durch Blutung im OP-Gebiet) die- nen, stellt die Blutsperre eine echte kausale Maßnahme dar, die das Koagulieren und Absaugen oft völlig entbehrlich macht.

Operationen mit Blutsperre bzw. in Blutleere kommen für die mittleren bis distalen Extremitäten infrage (es sei erwähnt, dass z.B. in der Abdominalchirurgie in Form des Pringle-Manövers ebenfalls dieses Prinzip angewendet wird). Proximal des OP-Gebietes wird z.B. eine Druckmanschette angelegt. Mit oder ohne vorheriges Ausstreichen oder Auswickeln der Extremität kann nach Schließen der Manschette oft nahezu bluttrocken operiert werden.

Eine weitere Indikation stellt die intravenöse Regionalanästhesie dar, bei der distal ein Lokalanästhetikum eingebracht wird, das durch die Blutsperre in der Extremität gehalten wird und so weitgehende Schmerzfreiheit ermöglicht. Im Vergleich zu anderen regionalanästhetischen Verfahren wie z.B. den Plexusblockaden sind die Anforderungen an den Anästhesisten hier deutlich geringer, was mit einer niedrigeren Versagerquote einhergeht.

\section{Kontraindikationen}

Anders als für die Hochfrequenzchirurgie und das Absaugen gibt es für die Anwendung von Blutsperren echte Kontraindikationen.

- Ausgeprägte Arteriosklerose/periphere AVK: die Anwendung einer Blutsperre ist aufgrund des hohen Risikos eines Gefäßverschlusses bzw. einer weiteren Verschlechterung der Durchblutungssituation kontraindiziert.

Präoperativ sollte bei jedem Patienten, v.a. bei Eingriffen an den Beinen, durch Erheben des Pulsstatus und ggf. die Bestimmung von arteriellen Verschlussdrücken die Durchblutungssituation überprüft werden. Im Verdacht sollte auf die Blutsperre verzichtet werden, da die Folgen vermehrter Blutungen viel besser beherrschbar sind als die eines Gefäßverschlusses.

- Infektion/septische Eingriffe: hier ist nicht unbedingt die Blutsperre, jedoch das vorherige Auswickeln kontraindiziert, da es zu einer Keimverschleppung kommen kann.

- Beim Auswickeln der Beine kann es zum Lösen einer vorbestehenden Beinvenenthrombose mit nachfolgender Embolie kommen. Daher wird an unserer Klinik an der unteren Extremität nicht ausgewickelt. 


\section{Funktionsweise}

An den Fingern reicht als Blutsperre ein Gummischlauch, der mit einer Klemme ne Tourniquets zum Einsatz.

An Oberarm und Oberschenkel werden in der Regel pneumatische, d.h. mit Druckluft betriebene, Manschetten verwendet, die im Prinzip wie eine Blutdruckmanschette aufgebaut sind (eine solche kann notfalls durchaus auch eingesetzt werden, hält aber den Druck kaum zuverlässig über eine längere Zeit und bietet keine gleichmäßige Druckverteilung).

Ähnlich dem Vakuum für Absauggeräte steht in Kliniken normalerweise Druckluft aus Wandanschlüssen zur Verfügung, die mit zwischengeschalteten Geräten reguliert wird. Ansonsten muss auf mobile Pumpen oder manuellen Betrieb zurückgegriffen werden.

\section{Praktischer Einsatz}

\section{Auswickeln}

Besonders bei Eingriffen an Hand und Unterarm kann durch Auswickeln der Extremität eine noch vollständigere Bluttrockenheit erzielt werden, da auch das venöse Blut entfernt wird. Dies lässt sich am besten durch das straffe Wickeln mit einer Gummibandage von distal nach proximal erreichen. Die Druckmanschette wird dann bei noch liegender Bandage aufgepumpt.

\section{Anlegen der Manschette}

Die Manschette sollte - typischerweise mit Verbandswatte - unterpolstert werden (Abb.5). Um Druckstellen und das fixiert wird, aus. Alternativ kommen klei-

Einklemmen von Hautfalten zu vermeiden, müssen Polsterung und Manschette straff und faltenfrei anliegen; die Verbandswatte dazu nicht zu dick wickeln: zwei Lagen reichen völlig aus.

Ein wesentliches Risiko für Hautschäden stellt unter die Manschette laufende Feuchtigkeit (besonders Desinfektionslösung) dar. Die Manschette sollte daher wenigstens nach distal, von wo am ehesten ein Eindringen $\mathrm{zu}$ befürchten ist abgedichtet werden. Am einfachsten ist dies mit den im OP sowieso vorhandenen wasserdichten Klebetüchern zu erreichen.

\section{Druck und Dauer}

Der Manschettendruck muss den Erfordernissen angepasst und sollte so niedrig wie möglich gewählt werden. Wesentliche Einflussgrößen sind: Umfang der Extremität, Weichteildeckung, Blutdruck (der durch die Anästhesie gesteuert möglichst nicht über $150-160 \mathrm{mmHg}$ systolisch liegen sollte) und Gefäßstatus des Patienten. Typische Werte für Erwachsene sind 250-300 mmHg an den Armen und 350-400 mmHg an den Beinen. Manche Autoren geben geringere Drücke als ausreichend an (z. B. S. Estersohn, Sourifman 1982). Es sind auch Geräte verfügbar, die den Manschettendruck automatisch an den Blutdruck des Patienten anpassen. Dann muss die gewünschte Differenz zum Blutdruck voreingestellt werden.

Als nicht $\mathrm{zu}$ überschreitende Dauer für die Blutsperre werden allgemein zwei Stunden angegeben. Auch hier gilt, dass man immer versuchen sollte, mit weniger auszukommen. Das Protokollieren der Zeitpunkte von Aufpumpen und Druck-

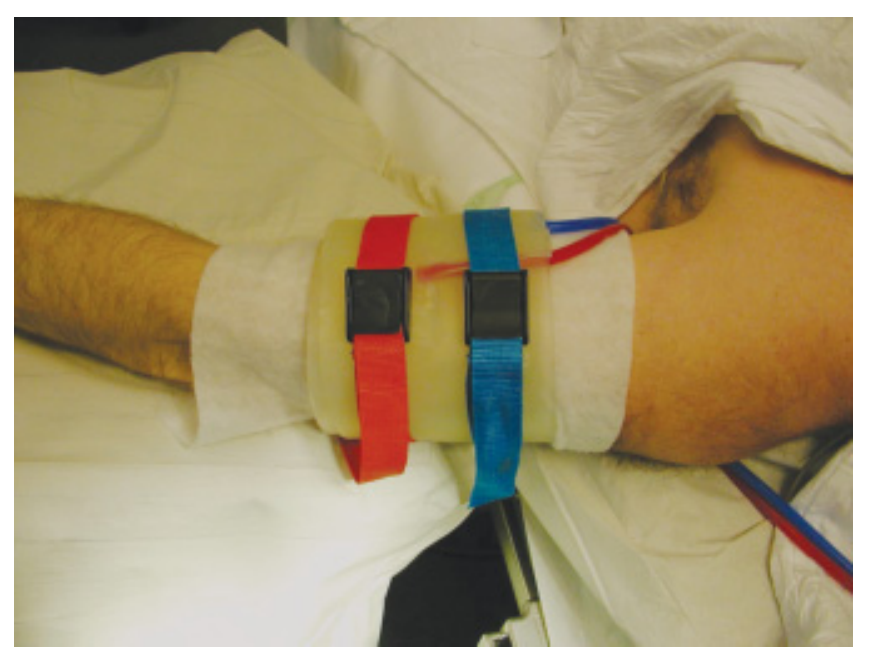

Abb. 5 Unterpolstern der Druck(l) einer dünnen (!) Lage Verbandswatte. ablassen sowie des angelegten Drucks muss Routine sein. Über die Effektivität eines zwischenzeitlichen Öffnens der Blutsperre besonders bei längeren Ischämiezeiten gibt es unterschiedliche Ansichten: von manchen Autoren wird es empfohlen, andere konnten tierexperimentell keinen positiven Effekt feststellen (z. B. Mohler et al. 1999).

\section{Komplikationen}

Schäden durch Druckmanschetten können langwierige und besonders kosmetisch sehr störende Folgen haben und geben immer wieder Anlass zu Schadenersatzforderungen. Es geht im Wesentlichen um zwei Gruppen von Schäden:

- Hautschäden, die das Bild einer Verbrennung oder Verätzung zeigen (durch Desinfektionslösung verursacht)

- Funktionelle Schäden an tiefer liegenden Strukturen, v.a. Gefäß- und Nervenschäden (durch zu hohen Druck verursacht).

Diese Komplikationen sind glücklicherweise eher selten; in der Literatur werden Werte von ca. 1/1000 für Hautschäden (Rudolph et al. 1990) und von zwischen 1/7000 und 1/4300 für Nervenschäden (Landi et al. 1995) angegeben. In nahezu allen Fällen ist mit einer Wiederherstellung der vollen Nervenfunktion zu rechnen; dies kann aber durchaus sechs bis neun Monate dauern.

Außer dem Beachten der oben genannten Kontraindikationen ist v.a. das regelrechte Anlegen und Abdichten der Manschette und das Beachten der Druck- und Zeitgrenzen entscheidend. Die Häufigkeit von Komplikationen sollte sich so deutlich minimieren lassen. Kann nachgewiesen werden, dass adäquat vorgegangen wurde, sind überdies Komplikationen nicht als Kunstfehler, sondern als nicht vermeidbare, verfahrenstypische Komplikationen anzusehen.

Blutsperren können zu verschiedenen ernsthaften Komplikationen führen, die sich bei sachgerechter Anwendung fast immer vermeiden lassen. 


\section{Danksagung}

Wir möchten der Firma Erbe in Tübingen für die Überlassung von Informationsmaterial zum Thema Hochfrequenzchirurgie danken

\section{Literatur}

1 Estersohn HS, Sourifman HA. The minimum effective midthigh tourniquet pressure. J Foot Surg 1982 Winter ; 21(4): 281-4

2 Mohler LR, Pedowitz RA, Myers RR et al.. Intermittent reperfusion fails to prevent posttourniquet neurapraxia. J Hand Surg [Am] 1999 Jul; 24(4): 687-93

${ }^{3}$ Rudolph H, Gärtner J, Studtmann V. Hautschäden nach Verwendung von Blutdruckmanschetten. Unfallchirurgie. 1990 Oct; 16(5): $244-51$

${ }^{4}$ Landi A, Saracino A, Pinelli M et al.. Tourniquet paralysis in microsurgery. Ann Acad Med Singapore 1995 Jul; 24(4 Suppl): 8993
Dr. med. Fabian Schäfer

Assistenzarzt

Prof. Dr. med. Jürgen Degreif

Chefarzt

Klinik für Unfallchirurgie und Orthopädie Städt. Kliniken Esslingen

Hirschlandstr. 97

D-73730 Esslingen 